\title{
Attitudes and perceptions of Dutch companion animal veterinarians towards antimicrobial use and antimicrobial resistance
}

\author{
Nonke E.M. Hopman ${ }^{\mathrm{a}}$, Lapo Mughini-Gras ${ }^{\mathrm{b}, \mathrm{c}}$, David C. Speksnijder ${ }^{\mathrm{a}, \mathrm{d}}$, Jaap A. Wagenaar ${ }^{\mathrm{a}, \mathrm{e}}$, \\ Ingeborg M. van Geijlswijk ${ }^{\mathrm{f}}$, Els M. Broens ${ }^{\mathrm{a}, *}$ \\ ${ }^{a}$ Department of Infectious Diseases and Immunology, Faculty of Veterinary Medicine, Utrecht University, Yalelaan 1, 3584 CL Utrecht, the Netherlands \\ ${ }^{\mathrm{b}}$ Institute for Risk Assessment Sciences (IRAS), Faculty of Veterinary Medicine, Utrecht University, Yalelaan 2, 3584 CL Utrecht, the Netherlands \\ ${ }^{\mathrm{c}}$ Center for Infectious Disease Control (CIb), National Institute for Public Health and the Environment (RIVM), Antonie van Leeuwenhoeklaan 9, 3721 MA Bilthoven, the \\ Netherlands \\ ${ }^{\mathrm{d}}$ University Farm Animal Clinic ULP, Reijerscopse Overgang 1, 3481 LZ Harmelen, the Netherlands \\ ${ }^{\mathrm{e}}$ Wageningen Bioveterinary Research, Houtribweg 39, 8221 RA Lelystad, the Netherlands \\ ${ }^{\mathrm{f}}$ Pharmacy Department, Faculty of Veterinary Medicine, Utrecht University, Yalelaan 106, 3584 CM Utrecht, the Netherlands
}

\section{A R T I C L E I N F O}

\section{Keywords:}

Antimicrobial use

Companion animal

Prescribing behaviour

Explanatory variable

Veterinary medicine

\begin{abstract}
A B S T R A C T
Antimicrobial use (AMU) in humans and animals facilitates the emergence of antimicrobial resistance (AMR). With increasing AMR being recognised as a major global threat for public health, responsible AMU is strongly advocated in both human and veterinary medicine. Knowledge on factors influencing antimicrobial prescribing behaviour of companion animal veterinarians is needed to promote responsible AMU in companion animals and to improve compliance with current legislation and guidelines. The present study aimed to quantitively investigate attitudes and perceptions of companion animal veterinarians towards AMU and AMR and to identify associations with demographic characteristics as possible explanatory variables. A self-administered questionnaire was developed based upon an earlier qualitative interview study, and 1608 potential participants (i.e. practising companion animal veterinarians) were invited. The questionnaire included questions addressing general descriptives of the respondents and questions with 6-point Likert scale statements, to assess attitudes towards AMU, AMR, factors influencing antimicrobial prescribing, and possible options to support responsible AMU.

The response rate was $32 \%(22 \%$ when complete questionnaires considered). Categorical Principal Component Analysis (CATPCA) was conducted on 76 Likert scale questions. This resulted in a final model with 37 questions explaining $38.7 \%$ of the variance of the question scores, with three underlying dimensions ("attitudinal profiles"). Additionally, general descriptives were added to the CATPCA as possible explanatory variables. The first dimension, related to "social responsibility" was positively associated with veterinarians working in clinics dedicated to companion animals, with veterinarians working in a referral clinic, and with more experienced veterinarians. The second dimension was related to "scepticism", which was positively associated with being a male veterinarian and with more experienced veterinarians. The third dimension was related to "risk avoidance", especially regarding surgical procedures, and was negatively associated with veterinarians working in clinics in urban areas and with veterinarians working part-time. Antimicrobial prescribing behaviour was self-reported to be well considered, and respondents did not see economic drivers as important influencing factors. The unwillingness of owners and financial constraints were perceived as important barriers for performing further diagnostics. To improve AMU, a multifaceted approach, taking differences between companion animal veterinarians (e.g., in experience and gender) and differences in work situation (e.g., full-time versus part-time) into account, should be directed at companion animal veterinarians and owners. Moreover, a joint and comprehensive effort of several stakeholders, like veterinary nurses, guideline developers, pharmaceutical industry, and providers of diagnostics, is needed to optimise AMU in companion animals.
\end{abstract}

\footnotetext{
* Corresponding author.

E-mail addresses: n.e.m.hopman@uu.nl (N.E.M. Hopman), lapo.mughini.gras@rivm.nl (L. Mughini-Gras), d.c.speksnijder@uu.nl (D.C. Speksnijder), j.wagenaar@uu.nl (J.A. Wagenaar), i.m.vangeijlswijk@uu.nl (I.M. van Geijlswijk), e.m.broens@uu.nl (E.M. Broens).
} 


\section{Introduction}

Antimicrobial use (AMU) in humans and animals facilitates the selection and dissemination of antimicrobial resistance (AMR) (EMA, 2013; Spellberg et al., 2013; McEwen and Collignon, 2018). AMR is a major global threat for public health; thus, responsible AMU is strongly advocated in both human and veterinary medicine (Collignon et al., 2016).

Since 2008, AMU in food-producing animals has received considerable attention in the Netherlands. Nationwide AMU-reducing action plans and several regulations have been implemented, resulting in an overall AMU reduction of almost 68\% during 2007-2017 (DoradoGarcia et al., 2016; NETHMAP/MARAN, 2018; Speksnijder et al., 2017). Since January 2013, Dutch legislation requires susceptibility testing prior to prescribing fluoroquinolones and 3rd and 4th generation cephalosporins. This holds for use in all animal species, including companion animals (STAATSCOURANT, 2013). Besides legislation, policies on veterinary AMU and guidelines on AMU in companion animals were developed (Working Party for Policy on Veterinary Antimicrobials; www.wvab.nl). Despite the fact that these guidelines are professional standards, uptake and implementation of these guidelines depends on the individual veterinarian. Research among foodproducing animal veterinarians has shown that attitudes and perceptions towards AMU and AMR have changed over the last years, partly because of more and stricter regulations and increased attention for the topic (Speksnijder et al., 2015; Coyne et al., 2016; Bourely et al., 2018). However, little is known about attitudes and perceptions of companion animal veterinarians towards AMU and AMR. Knowledge on factors influencing antimicrobial prescribing behaviour of companion animal veterinarians is needed to promote responsible AMU in companion animals and to improve compliance to current legislation and guidelines. Some qualitative research in companion animals has been done, mainly in the UK (Mateus et al., 2014; King et al., 2018; Smith et al., 2018). In 2015, a qualitative study among 18 Dutch companion animal veterinarians was performed to explore factors influencing antimicrobial prescribing behaviour (Hopman et al., 2018). The conceptual model of this qualitative study showed four major groups of influencing factors on antimicrobial prescribing: veterinarian-related factors, patient-related factors (i.e. owner- and pet-related), treatment-related factors (i.e. non-antimicrobial treatment options and antimicrobial-related factors), and contextual factors (i.e. professional interactions, further diagnostics and environmental factors). The present study aimed to study these factors in a quantitative way among Dutch companion animal veterinarians by investigating their attitudes and perceptions towards AMU and AMR. A second aim was to identify possible associations between these attitudes and perceptions, and demographic characteristics as possible explanatory variables. The results of this study will be used to provide input for the development and implementation of an antimicrobial stewardship programme in Dutch companion animal clinics.

\section{Material and methods}

\subsection{Study materials}

A self-administered questionnaire was developed based on the results of an earlier qualitative study (Hopman et al., 2018). The questionnaire was divided into three parts: 1) 16 questions addressing general descriptives and demographics, 2) 76 questions with 6-point Likert scale statements $(1=$ completely disagree; $2=$ disagree; $3=$ tend to disagree; $4=$ tend to agree; $5=$ agree; $6=$ completely agree) to assess attitudes towards AMU, including factors influencing antimicrobial prescribing behaviour and perceptions on AMR and responsible AMU, and 3) 14 6-point Likert scale questions related to possible options to support responsible AMU. In one of the questions from part 1 respondents were asked whether they perceived the clinic they worked in as an urban or rural clinic. For some questions, the option 'not applicable' was added. The design and content of the questionnaire was discussed in detail with experts from human and veterinary medicine and the questionnaire was subsequently piloted amongst veterinarians working in the field and veterinarians working at the Faculty of Veterinary Medicine of Utrecht University. The questionnaire was administered online using SurveyMonkey (SurveyMonkey Inc., San Mateo, California USA, www.surveymonkey.com).

\subsection{Study population}

The exact number of Dutch veterinarians working with companion animals was estimated to be between 1800 and 2000 at the time of this study (August 2015, personal communication). In total, 1608 unique email addresses of companion animal veterinarians were obtained from the Royal Netherlands Veterinary Association (KNMvD) and the Collective of Practising Veterinarians (CPD) for potential enrolment in the study. (KNMvD and CPD are the two major veterinary professional associations in the Netherlands, representing the majority of Dutch veterinarians). This list was not a perfect and complete list. The list contained duplicates (e.g., private and work email address of the same veterinarian), as well as disused email addresses, and email addresses of veterinarians who were not practising anymore. Email addresses of some companion animal veterinarians were obviously missing.

In September 2015, an invitation to participate in the online survey was sent. After 3 and 8 weeks, a reminder was sent to non-responders. The survey was also advertised in newsletters of the KNMvD and CPD. Responses were collected anonymously unless participants voluntarily chose to leave their contact details. A $€ 50$ voucher was allotted as a financial incentive to one out of every 50 respondents completing the questionnaire. All returned questionnaires were handled confidentially.

\subsection{Data analysis}

As the aim was to focus on companion animal veterinarians, only respondents who stated that they currently work in a practice and spend more than $50 \%$ of their working hours on companion animals were included in the survey.

The 6-point Likert scale questions were described using mean values, mode values, and standard deviations. Mean values, in particular, were used to quantify the central tendency of questions to which most respondents disagreed or agreed. A Categorical Principal Component Analysis (CATPCA) was performed as described in detail by Speksnijder et al. (2015), to reduce the attitudinal variables (i.e. the single Likert scale questions) to several uncorrelated principal components (dimensions), which reflected the information in the original data. The uncorrelated principal components were further analysed to assess differences in attitudes according to veterinarian's demographics (Linting and van der Kooij, 2012; Speksnijder et al., 2015). CATPCA was used because it can manage possibly nonlinearly related variables with different types of measurement levels and is particularly useful to analyse Likert-type variables.

The 76 Likert scale questions from part 2 were included in the CATPCA. For missing values, the default Passive CATPCA option of imputing the modal category after quantification was chosen. Scree plot analysis indicated that a 3-dimension solution was most suitable for analysis of the whole dataset (of originally 76 questions). All variables (i.e. individual Likert scale questions) with a total Variance Accounted For (VAF) of 0.25 or lower were excluded from the final analysis. The CATPCA procedure was repeated until no variables with a total VAF $<0.25$ remained. Subsequently, component loadings were calculated of which loadings of 0.40 or higher were regarded as sufficient to calculate object scores for each dimension and these were used for further analysis. Based on the component loadings, resulting dimensions were then interpreted as "attitudinal profiles" (Linting and van der Kooij, 2012; Speksnijder et al., 2015). Finally, the association 
between the explanatory variables from part 1 (i.e. demographics of the respondents) and each separate dimension was assessed using linear regression analysis, first univariately (each explanatory variable at a time) and then multivariately. Only those variables with a p-value < 0.10 in the univariate analysis were included in the multivariate models, which were reduced in a backward stepwise fashion until only variables with a p-value $<0.05$ remained. Variables that changed the effect of the other covariates by $>10 \%$ when removed from the model were considered as possible confounders and therefore retained in the model.

Data were analysed using Microsoft Excel, IBM SPSS Statistics (version 24) and STATA (version 15).

\section{Results}

\subsection{General descriptives}

Questionnaires were received from 508 (32\%) respondents. Of these, 89 veterinarians indicated in part 1 of the questionnaire that they do not currently work in a companion animal practice or spend less than $50 \%$ of their working hours on companion animals. They were therefore excluded. 353 respondents completed part 1 and 2 of the questionnaire, and 350 respondents completed part 3 as well, resulting in a response rate of $22 \%$ (based on the 1608 email addresses used). The demographics of the 353 respondents who completed part 1 and 2 of the questionnaire are shown in Table 1.

\subsection{Behaviour, attitudes and perceptions}

Based on the Likert scale scores $(1=$ completely disagree to $6=$ completely agree), the 5 questions with a mean score $<2$ (i.e. majority of respondents disagreed) and the 11 questions with a mean

Table 1

Demographics of respondents who completed parts 1 and 2 of the questionnaire $(\mathrm{n}=353)$.

\begin{tabular}{|c|c|}
\hline Demographic & Number (\%) \\
\hline \multicolumn{2}{|c|}{ Percentage of working hours spent on companion animals: } \\
\hline $50-75 \%$ & $16(4.5)$ \\
\hline$>75 \%$ & $337(95.5)$ \\
\hline \multicolumn{2}{|l|}{ Gender: } \\
\hline Male & $134(38)$ \\
\hline Female & $219(62)$ \\
\hline \multicolumn{2}{|l|}{ Type of clinic (1): } \\
\hline Mixed-animals & $96(27.2)$ \\
\hline Companion animals only & $257(72.8)$ \\
\hline \multicolumn{2}{|l|}{ Type of clinic (2): } \\
\hline Rural & $143(40.5)$ \\
\hline Urban & $210(59.5)$ \\
\hline \multicolumn{2}{|l|}{ Work situation (1): } \\
\hline Clinic owner & $202(57.2)$ \\
\hline Working on payroll & $132(37.4)$ \\
\hline Other & $19(5.4)$ \\
\hline \multicolumn{2}{|l|}{ Work situation (2) : } \\
\hline Full-time & $184(52.1)$ \\
\hline Part-time & 169 (47.9) \\
\hline \multicolumn{2}{|l|}{ Work situation (3): } \\
\hline First opinion & $320(90.7)$ \\
\hline Referral & $14(4.0)$ \\
\hline \multirow[t]{2}{*}{ Other } & $19(5.4)$ \\
\hline & Median (min-max) \\
\hline Year of birth & 1969 (1944-1990) \\
\hline Year of graduation & 1997 (1966-2014) \\
\hline Working experience (years) & $(1-46)$ \\
\hline
\end{tabular}

* Based upon the respondent's own perception of rural versus urban and fulltime versus part-time. score $>5$ (i.e. majority of respondents agreed) are displayed in Table 2, as well as all those questions that could be considered as possible barriers to responsible AMU with a mean score $>4$. The questions with a mean score $>5$ and $<2$ show that respondents almost unanimously report their decision-making in antimicrobial prescribing as well considered and not influenced by opinions or pressure from clients and colleagues. The majority of respondents seem to be aware of AMR and practice policies are supportive in using AMs responsibly. With regard to the performance of further diagnostics, reluctance of companion animal owners and their (possibly related) financial constraints were mentioned as possible barriers.

\subsection{3-dimensional CATPCA}

The CATPCA resulted in a 3-dimensional solution with 37 variables. The 3-dimensional CATPCA explained $38.7 \%$ of the variance of the scores provided by the respondents. Component loadings (as shown in Table 3) for the 3-dimensional solution are correlations (either positively or negatively) between the single Likert scale questions and the dimensions.

Based on the grouping of different variables that have high-value loadings on the different dimensions, three dimensions (latent variables) can be described:

- Dimension 1 is related to "social responsibility", well-considered antimicrobial prescribing, self-confidence, independence and recognition of the authority/role of the veterinarian in public and animal health, which is not easily influenced by owner's demands and is related to working in a well-equipped clinic.

- Dimension 2 is related to "scepticism" as reflected in "no harm done by trying antimicrobials", risk avoidance related to the individual animal and ignorance of the possible (public health) risks of AMU in companion animals as related to AMR emergence in companion animals, and in general.

- Dimension 3 is related to fear of the possible consequences of not prescribing antimicrobials, a "better safe than sorry" habit, mainly related to possible infections after surgical procedures.

\subsubsection{Regression analysis of demographics on CATPCA dimensions}

The results of the univariate and multivariate analysis assessing the association between demographics with the above-defined three dimensions are shown in Tables 4 and 5 .

Results from the multivariate regression analysis show that dimension 1 ("social responsibility") is positively associated with veterinarians working in clinics entirely dedicated to companion animals, with veterinarians working in a referral clinic, and with more experienced veterinarians (i.e. working more years in practice). Dimension 2 ("scepticism") is positively associated with being a male veterinarian and with more experienced veterinarians. Dimension 3 ("risk avoidance") is negatively associated with veterinarians working in clinics in urban areas and with veterinarians working part-time.

\subsection{Possible options to support responsible AMU}

The 14 questions in part 3 of the questionnaire on possible options to support responsible AMU were scored on a 6-point Likert scale by 350 veterinarians (3/353 veterinarians did not complete part 3 of the questionnaire). These questions and their mean scores are shown in Fig. 1, which gives a general impression on how these possible options to support responsible AMU are perceived.

Promising options to support responsible AMU (Fig. 1) seem to be the encouragement of supportive treatment options not containing antimicrobials. More education on responsible AMU for veterinarians and education of companion animal owners on AMR and responsible AMU also scored relatively high. Decoupling of prescribing and selling antimicrobials by veterinarians scored lowest. 
Table 2

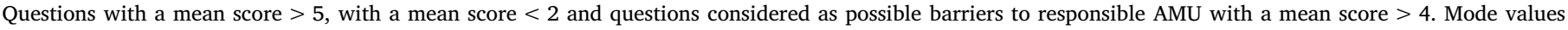

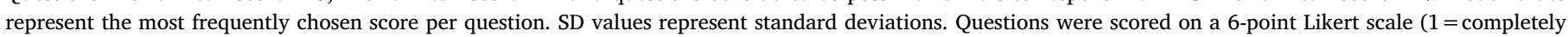
disagree; $6=$ completely agree).

\begin{tabular}{|c|c|c|c|}
\hline Questions & Mean & Mode & SD \\
\hline \multicolumn{4}{|l|}{ With a mean score $>5$} \\
\hline My choice for a specific type of $\mathrm{AM}^{*}$ has nothing to do with higher financial profits. & 5.49 & 6 & 1.075 \\
\hline My choice for a specific type of AM has nothing to do with acquiring more clients. & 5.49 & 6 & 1.036 \\
\hline In my clinic, we have sufficient possibilities to send in samples to a laboratory for culture and susceptibility tests. & 5.39 & 5 & 0.645 \\
\hline My choice for a specific type of AM is regardless of my perception of what an owner wants. & 5.24 & 5 & 0.806 \\
\hline I am okay with the increased attention on AMU in companion animals. & 5.21 & 5 & 0.874 \\
\hline My choice for a specific type of AM has nothing to do with what an owner wants. & 5.15 & 5 & 0.853 \\
\hline I think twice before I prescribe AMs. & 5.14 & 5 & 0.647 \\
\hline In my clinic, sufficient possibilities to perform further diagnostics (other than culture and susceptibility tests) are available. & 5.13 & 5 & 0.852 \\
\hline When choosing a specific type of AM, I consider which pathogens might be involved. & 5.08 & 5 & 0.672 \\
\hline My veterinary colleague(s) and I support each other to show restraint in prescribing AMs. & 5.06 & 5 & 1.037 \\
\hline Our practice policy is committed to showing restraint in prescribing AMs. & 5.05 & 5 & 1.082 \\
\hline \multicolumn{4}{|l|}{ With a mean score $<2$} \\
\hline Because a neighbouring clinic is easy in prescribing AMs, I tend to do so as well. & 1.97 & 2 & 0.9 \\
\hline After most surgical procedures, I habitually prescribe AMs. & 1.90 & 1 & 1.13 \\
\hline Using an $\mathrm{AM}$ is fine as long as it causes no harm. & 1.69 & 1 & 0.825 \\
\hline I quite often experience pressure from colleagues or superiors to prescribe specific types of AMs I disagree with. & 1.66 & 1 & 1.001 \\
\hline After elective surgery (neuter/spay) without prescribing AMs the risk of complications is too high. & 1.61 & 1 & 0.923 \\
\hline \multicolumn{4}{|l|}{ Considered as possible barriers to responsible AMU, with a mean score $>4$} \\
\hline An important hurdle to performing further diagnostics (including culture and sensitivity tests) is that owners do not want to pay for it. & 4.55 & 5 & 1.107 \\
\hline After negative experiences with a specific type of AM, I tend not to use that type of AM again. & 4.06 & 4 & 1.084 \\
\hline I regularly encounter companion animal owners urging to try AMs first before performing further diagnostics. & 4.04 & 5 & 1.355 \\
\hline
\end{tabular}

* $\mathrm{AM}=$ antimicrobial.

\section{Discussion}

This study showed different attitudes and perceptions of Dutch companion animal veterinarians towards AMU and AMR and revealed associations with demographic characteristics.

Whereas financial pressure or the tendency to meet client's expectations have been identified as possible drivers of inappropriate AMU in other studies (Mateus et al., 2014; Speksnijder et al., 2015; Smith et al., 2018; Hopman et al., 2018), veterinarians in our survey reported that their antimicrobial prescribing behaviour was hardly influenced by economic drivers nor by owners' demands. This indicates an autonomous working routine, although this might be biased by the fact that in our study it concerns self-reported behaviour, based upon own opinions and views. Therefore, it is possible that veterinarians did not want to openly admit that owners or economic drivers might influence their antimicrobial prescribing behaviour. On the other hand, campaigns on AMR aimed at the general public might have resulted in increased awareness among companion animal owners and with that decreased pressure to prescribe antimicrobials. Furthermore, the implementation of guidelines and legislation on veterinary AMU in recent years might act as a supportive tool for veterinarians to convince companion animal owners and to withstand their strong demands (Hopman et al., 2018).

Respondents reported to have sufficient possibilities in their clinics to perform further diagnostics and to send in samples for susceptibility testing. However, unwillingness of an owner and financial constraints are indicated as possible barriers to perform further diagnostics. Other studies support these findings (Mateus et al., 2014; Jessen et al., 2017; Hardefeldt et al., 2018a; Bourely et al., 2018). Development of cheaper and faster diagnostics will increase the use of diagnostic testing and support responsible antimicrobial prescribing (De Briyne et al., 2013; Bourely et al., 2018).

In the assessment of the possible options to support responsible AMU (Fig. 1), options regarding "decoupling" of antimicrobial sales and prescribing scored lowest. This finding is supported by other studies on antimicrobial prescribing in food-producing animals (Speksnijder et al., 2015; Coyne et al., 2016). A possible explanation of why this option was less favourable could be fear to lose profit. On the other hand, veterinarians reported their antimicrobial prescribing behaviour to be independent of economic drivers. The fear to lose the right to sell antimicrobials might also originate from a fear of losing autonomy, additional administrative procedures, time delays between prescribing and actual administration of antimicrobials, and practical disadvantages for companion animal owners.

The CATPCA revealed three main attitudinal profiles, and differences in these profiles were associated with several demographic characteristics. Attitudes, such as "social responsibility" and acting selfconfident and independently (the first dimension), were positively associated with more experienced veterinarians and veterinarians dedicated to treating companion animals. Several studies showed that younger veterinarians might experience more difficulties in acting independently from (perceived) demands of animal owners (Speksnijder et al., 2015; Bourely et al., 2018), implying that younger veterinarians might be less self-confident. The positive association with veterinarians dedicated to treating companion animals only could be the result of better knowledge or awareness of new treatment options and specific guidelines.

Scepticism about the possible risks of AMU in companion animals as related to AMR emergence in companion animals and general healthcare was positively associated with males and more experienced veterinarians. The study of Speksnijder et al. (2015) supports this finding stating that increased experience is associated with being less concerned about a possible veterinary contribution to AMR. When optimising AMU in all Dutch companion animal clinics, these differences in knowledge and attitudes on responsible AMU and the importance of AMR between less and more experienced veterinarians should be taken into account. This could be done, for example, by offering educational training on the latest insights on AMR to more experienced veterinarians or training on communication skills to less experienced veterinarians.

Working in rural areas and working full-time were positively associated with risk avoidance, especially regarding surgical procedures. Hardefeldt et al. (2017) did not find a difference between rural and metropolitan clinics when comparing compliance with AIDAP (Australian Infectious Disease Advisory Panel) and BSAVA (British Small Animal Veterinary Association) guidelines on AMU for surgical 
Table 3

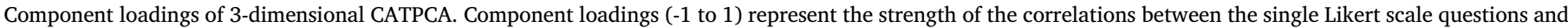

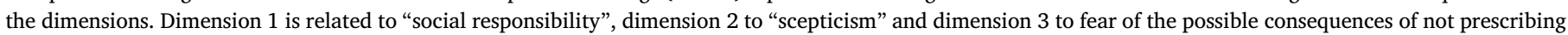
AMs.

Questions

\begin{tabular}{lll}
\multicolumn{2}{l}{ Dimension } & \\
\hline 1 & 2 & 3 \\
\hline & -0.546 & \\
& & 0.678 \\
-0.478 & & 0.607 \\
0.502 & & -0.486
\end{tabular}

There is enough evidence proving that alternative treatment options (i.e. non-AM treatment options) are as effective as treatments with AMs.

Before most surgical procedures, I usually give an AM injection.

After most surgical procedures, I habitually prescribe AMs.

During standard surgery procedures (neuter and spay), I always work meticulously, and I have a clean operating theatre, which means AMs are redundant.

When choosing a specific type of AM, I take possible adverse events into account.

I do not await the results of culture and susceptibility tests or further diagnostics to prescribe AMs, because I think the risk of medical complications is too high.

When I know a dog or cat has a comorbidity (e.g. diabetes mellitus), I am more inclined to prescribe third choice AMs.

In general, the risk of complications because of NOT prescribing AMs is bigger than the risk of problems with AMR because of prescribing AMs.

After elective surgery (neuter/spay) without prescribing AMs the risk of complications is too high.

Our practice policy is committed to showing restraint in prescribing AMs.

The risk of a resistant bacterium spreading as a result of my professional habits is very small.

The risk that a specific type of AM that I prescribe can no longer be used in human medicine in the future due to AMR is small.

I am okay with the increased attention on AMU in companion animals.

There is little research proving that AMU in companion animals contributes to problems with AMR in animals.

There is little research proving that AMU in companion animals contributes to problems with AMR in humans.

My veterinary colleague(s) and I support each other to show restraint in prescribing AMs.

I think twice before I prescribe AMs.

For every single patient, I deliberately choose which type of AM to prescribe.

In the case of a non-cooperative animal, I am more inclined to prescribe long-acting injectable AMs.

When prescribing AMs, it is important to use (results of) bacterial cultures and susceptibility tests.

It is easy to translate culture and susceptibility results into practical applications.

It takes too long before I receive my results of further diagnostics (other than culture and susceptibility tests).

When prescribing AMs, it is important to use further diagnostics (other than culture and susceptibility tests, e.g. diagnostic imaging or urine analysis).

Further diagnostics (other than culture and susceptibility tests) help me in choosing a specific type of AM.

In my clinic, we have sufficient possibilities to send in samples to a laboratory for culture and susceptibility tests.

In my clinic, sufficient possibilities to perform further diagnostics (other than culture and susceptibility tests) are available.

It is important to have a clear diagnosis before prescribing AMs.

I regularly have practical problems in taking samples for culture and susceptibility tests.

When choosing a specific type of AM, I take into account whether it is a bactericidal or -static AM.

When choosing a specific type of AM, I take my knowledge about pharmacokinetics into account.

When choosing a specific type of AM, I consider which pathogens might be involved.

My choice for a specific type of AM is regardless of my perception of what an owner wants.

I find it difficult NOT to prescribe AMs when an owner wants me to prescribe AMs.

My choice for a specific type of AM has nothing to do with what an owner wants.

I think it is important that the AM I prescribe is authorised for the specific indication and animal species concerned.

Even when a shift is busy, I take enough time to apply alternative treatment options (i.e. non-AM treatment options).

Practically performing further diagnostics is too time-consuming (including culture and sensitivity tests).

Total (Eigenvalue)

Cronbach's Alpha

VAF\%

0.487

$$
0.52
$$

$-0.516$

$-0.465$

0.518

0.62

0.645

0.648

0.648
-0.531

0.692

0.742

0.481

0.534

0.572

$-0.44$

0.446

0.56

$-0.543$

0.471

0.603

0.618

0.549

$-0.593$

0.429

0.442

0.555

0.578

$-0.445$

0.555

0.481

0.573

$-0.536$

7.692

0.894

$20.80 \%$
$3.881 \quad 2.727$

$0.763 \quad 0.651$

$10.50 \% \quad 7.40 \%$

$* \mathrm{AM}=$ antimicrobial.

prophylaxis. However, they found that the odds of compliance was 1.4 (95\% CI, 1.1-1.9) times greater for companion animal veterinarians compared to mixed species veterinarians. In another study, Hardefeldt et al. (2018b) found that animals from urban areas had 35\% higher odds of having an insurance claim submitted and a $6.3 \%$ higher odds of having AMs prescribed compared to animals from rural areas, which seems to be contrary to the findings of our present study. The difference could be the result of differences in attitudes of companion animal owners in urban versus rural areas, e.g., with regard to when they seek for veterinary care. The difference might also be explained by the lack of a clear definition of urban and rural. In both studies, the distinction was based upon the opinion of responding veterinarians and not on a clear definition or postal code. A possible explanation why full-time working veterinarians in rural areas were positively associated with this behavioural profile of "risk avoidance" (e.g., unnecessary surgical prophylaxis) could be differences in clinic policies, facilities and equipment, and different client expectations in comparison with urban clinics. In the past, rural clinics mainly focused on food-producing animals, but now they are focussing more on companion animals too. The facilities, equipment and clinic policies might be somehow different from typical urban clinics, in which the focus has always been on companion animals. Besides, companion animal owners in rural areas might have other (financial) expectations than companion animal owners in cities. To increase adherence to current guidelines and to decrease unnecessary AMU, more attention should be paid to education on hygiene measures and AM prophylaxis around surgery (e.g., no standard AMU as prophylaxis for routine surgery), and veterinarians' fears to omit AMU should be further explored, especially in rural clinics.

This study has some strengths and limitations that need to be addressed. The relatively low response rate of $32 \%$ (22\% based on complete questionnaires), although comparable with other studies (Speksnijder et al., 2015; Jessen et al., 2017; Hardefeldt et al., 2017), might have caused participation bias. Regarding mean age and mean age at graduation of the respondents, as well as the proportion of female and male respondents and their provinces of employment, the demographics were comparable to those of the Dutch population of veterinarians (Jaarsma et al., 2008; personal communication from Speksnijder et al., 2015; KNMvD, 2013).

Due to the study design, socially desirable answers to the 
Table 4

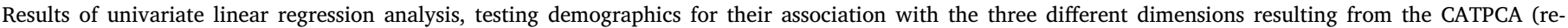

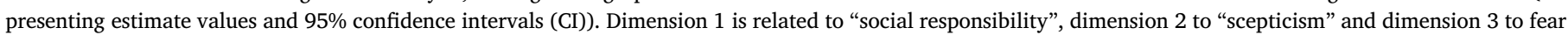
of the possible consequences of not prescribing AMs.

\begin{tabular}{|c|c|c|c|}
\hline Univariate & $\begin{array}{l}\text { Dimension } 1 \\
\text { Estimate }(95 \% \mathrm{CI})\end{array}$ & $\begin{array}{l}\text { Dimension } 2 \\
\text { Estimate }(95 \% \mathrm{CI})\end{array}$ & $\begin{array}{l}\text { Dimension } 3 \\
\text { Estimate }(95 \% \mathrm{CI})\end{array}$ \\
\hline $\begin{array}{l}\text { Percentage of working hours spend on companion animals ( }>75 \% \text { versus } 50-75 \% \text { ) } \\
\text { Gender (male versus female) }\end{array}$ & $\begin{array}{l}0.49(-0.01 \text { to } 1.00) \\
0.23(0.02 \text { to } 0.45)^{*}\end{array}$ & $0.62(0.41 \text { to } 0.82)^{*}$ & $-0.43(-0.93$ to 0.07$)$ \\
\hline $\begin{array}{l}\text { Type of clinic (1) (companion animals only versus mixed-animals) } \\
\text { Type of clinic (2) (urban versus rural) }\end{array}$ & $\begin{array}{l}0.35(0.12 \text { to } 0.58)^{*} \\
0.22(0.01 \text { to } 0.44)^{*}\end{array}$ & & $\begin{array}{l}-0.22(-0.46 \text { to } 0.01) \\
-0.23(-0.44 \text { to }-0.02)^{*}\end{array}$ \\
\hline $\begin{array}{l}\text { Work situation (1) (working on payroll versus clinic owner) } \\
\text { Work situation (1) (other versus clinic owner) } \\
\text { Work situation (2) (part-time versus full-time) } \\
\text { Work situation (3) (referral versus first opinion) } \\
\text { Work situation (3) (faculty versus first opinion) } \\
\text { Work situation (3) (other versus first opinion) }\end{array}$ & $\begin{array}{l}-0.35(-0.57 \text { to }-0.13)^{*} \\
-0.09(-0.56 \text { to } 0.38) \\
-0.26(-0.47 \text { to }-0.05)^{*} \\
1.20(0.68 \text { to } 1.72)^{*} \\
-0.02(-1.37 \text { to } 1.33) \\
0.80(0.33 \text { to } 1.28)^{*}\end{array}$ & $\begin{array}{l}-0.40(-0.61 \text { to }-0.18)^{*} \\
-0.26(-0.73 \text { to } 0.21) \\
-0.42(-0.62 \text { to }-0.21)^{*}\end{array}$ & $\begin{array}{l}-0.25(-0.46 \text { to }-0.04)^{*} \\
0.53(-0.00 \text { to } 1.07) \\
-0.75(-2.14 \text { to } 0.64) \\
0.48(-0.01 \text { to } 0.97)\end{array}$ \\
\hline $\begin{array}{l}\text { Year of birth } \\
\text { Year of graduation } \\
\text { Work experience (per year) }^{* * *}\end{array}$ & $\begin{array}{l}-0.01(-0.02 \text { to } 0.00) \\
-0.01(-0.02 \text { to } 0.00)^{*} \\
0.01(0.00 \text { to } 0.02)^{*}\end{array}$ & $\begin{array}{l}-0.02(-0.03 \text { to }-0.01)^{*} \\
-0.02(-0.03 \text { to }-0.01)^{*} \\
0.03(0.02 \text { to } 0.04)^{*}\end{array}$ & \\
\hline $\begin{array}{l}\text { Number of veterinarians per clinic only treating companion animals } \\
\text { Number of veterinarians per clinic also treating other species }\end{array}$ & $\begin{array}{l}0.04(0.01 \text { to } 0.07) \\
-0.04(-0.08 \text { to } 0.01)\end{array}$ & & \\
\hline
\end{tabular}

* p-value $<0.05$.

$* *$ Years as continous variable.

questionnaire might have been received. However, respondents had the opportunity to return the questionnaire anonymously (approximately $40 \%$ of the completed questionnaires), minimising this potential bias. Moreover, answers in the survey differed considerably between respondents, and all scores (totally disagree to totally agree) were chosen. This indicates that these data support a diversity of opinions and attitudes regarding AMU and AMR among Dutch companion animal veterinarians.

In conclusion, self-reported antimicrobial prescribing behaviour among companion animal veterinarians in the Netherlands appears to be well considered and not influenced by economic drivers. Unwillingness of owners and financial constraints were perceived as important barriers for performing further diagnostics. Changing prescribing behaviour is complex and we recommend that measures or strategies to improve AMU are diverse, multimodal and attuned to the specific situation (Hulscher et al., 2010; Smith et al., 2018; Hopman et al., 2018; Currie et al., 2018). Results of the present study will be used in the development of an antimicrobial stewardship programme (ASP) in Dutch companion animal clinics. A multifaceted and dynamic approach will be applied to safeguard the clinical efficacy of antimicrobials by optimising AMU while minimising the emergence of AMR and other possible adverse effects (Guardabassi and Prescott, 2015; Prescott and Boerlin, 2016). This multifaceted approach, taking differences between companion animal veterinarians (e.g., in experience and gender) and differences in work situation (e.g., urban versus rural clinics) into account, should not only be directed at companion animal veterinarians but also companion animal owners. Educational training and peer-to-peer consultation on AMU, AMR, the implementation and use of current guidelines and legislation should be included in this ASP. Other stakeholders, such as veterinary nurses, guideline developers, pharmaceutical industry, and providers of diagnostics, should be involved as well to stimulate their input in a joint and comprehensive effort to optimise AMU in companion animals. This could be done, for example, by informing them on the outcomes of present study and by organising a stakeholders meeting.

\section{Funding}

This study was funded by ZonMw (Netherlands Organisation for Health Research and Development, The Hague, the Netherlands), project number 205300003.

\section{Submission declaration}

This research study has not been published elsewhere previously.

Table 5

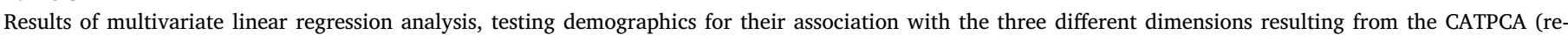

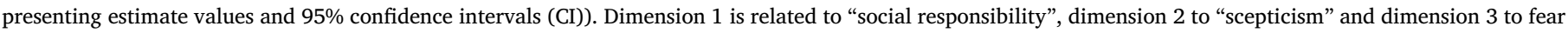
of the possible consequences of not prescribing AMs.

\begin{tabular}{|c|c|c|c|}
\hline Multivariate & $\begin{array}{l}\text { Dimension } 1 \\
\text { Estimate }(95 \% \mathrm{CI})\end{array}$ & $\begin{array}{l}\text { Dimension } 2 \\
\text { Estimate }(95 \% \mathrm{CI})\end{array}$ & $\begin{array}{l}\text { Dimension } 3 \\
\text { Estimate }(95 \% \mathrm{CI})\end{array}$ \\
\hline Gender (male versus female) & & $0.50(0.28 \text { to } 0.72)^{*}$ & \\
\hline Type of clinic (1) (companion animals only versus mixed-animals) & $0.28(0.06 \text { to } 0.51)^{*}$ & & \\
\hline Type of clinic (2) (urban versus rural) & & & $-0.22(-0.43 \text { to }-0.01)^{*}$ \\
\hline Work situation (2) (part-time versus full-time) & & & $-0.24(-0.45$ to -0.03$)$ \\
\hline Work situation (3) (referral versus first opinion) & $1.14(0.63 \text { to } 1.66)^{*}$ & & \\
\hline Work situation (3) (faculty versus first opinion) & $-0.15(-1.47$ to 1.17$)$ & & \\
\hline Work situation (3) (other versus first opinion) & $0.75(0.28 \text { to } 1.21)^{*}$ & & \\
\hline Work experience (per year) & $0.01(0.00 \text { to } 0.02)^{*}$ & $0.02(0.01 \text { to } 0.03)^{*}$ & \\
\hline
\end{tabular}

* p-value $<0.05$.

** Years as continous variable. 


\section{Possible options to support responsible AMU}

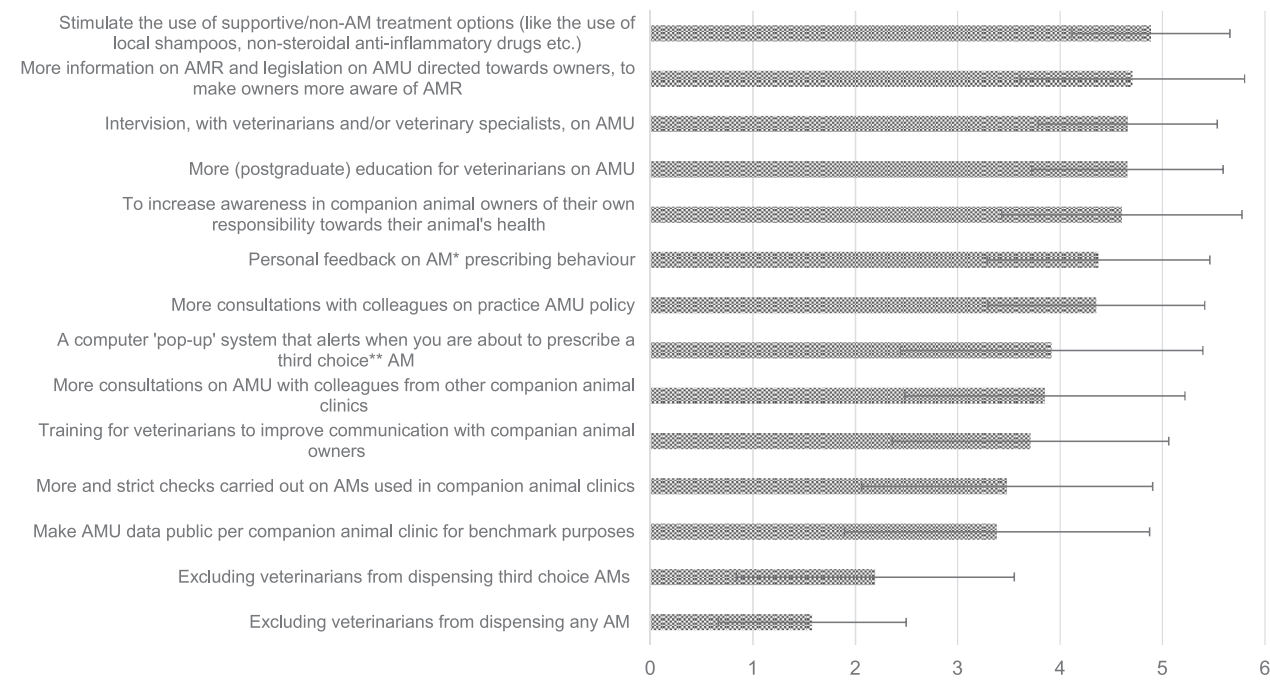

Fig. 1. Questions on possible options to support responsible AMU. Questions were scored on a 6 -point Likert scale $(1=$ completely disagree; $6=$ completely agree $)$ and are displayed here in order of highest to lowest mean score.

\section{Acknowledgements}

We are very grateful to all respondents for their participation in this study. We especially thank M.E.J.L. Hulscher for her help in developing the questionnaire and the remaining members of the ASAP project group (J.T. Bosje, D.J.J. Heederik, L.J. Hellebrekers, M.F.M. Langelaar, J.M. Prins, L. Schipper, and T.J.M. Verheij) for their input. We kindly acknowledge those veterinarians who critically evaluated and pretested the questionnaire.

\section{References}

Bourely, C., Fortane, N., Calavas, D., Leblond, A., Gay, E., 2018. Why do veterinarians ask for antimicrobial susceptibility testing? A qualitative study exploring determinants and evaluating the impact of antibiotic reduction policy. Prev. Vet. Med. 159, $123-134$.

Collignon, P.C., Conly, J.M., Andremont, A., McEwen, S.A., Aidara-Kane, A., World Health Organization Advisory Group, Bogota Meeting on Integrated Surveillance of Antimicrobial Resistance (WHO-AGISAR), Agerso, Y., Andremont, A., Collignon, P., Conly, J., Dang Ninh, T., Donado-Godoy, P., Fedorka-Cray, P., Fernandez, H., Galas, M., Irwin, R., Karp, B., Matar, G., McDermott, P., McEwen, S., Mitema, E., ReidSmith, R., Scott, H.M., Singh, R., DeWaal, C.S., Stelling, J., Toleman, M., Watanabe, H., Woo, G.J., 2016. World health organization ranking of antimicrobials according to their importance in human medicine: a critical step for developing risk management strategies to control antimicrobial resistance from food animal production. Clin. Infect. Dis. 63, 1087-1093.

Coyne, L.A., Latham, S.M., Williams, N.J., Dawson, S., Donald, I.J., Pearson, R.B., Smith, R.F., Pinchbeck, G.L., 2016. Understanding the culture of antimicrobial prescribing in agriculture: a qualitative study of UK pig veterinary surgeons. J. Antimicrob. Chemother. 71, 3300-3312.

Currie, K., King, C., Nuttall, T., Smith, M., Flowers, P., 2018. Expert consensus regarding drivers of antimicrobial stewardship in companion animal veterinary practice: a Delphi study. Vet. Rec. 182, 691.

De Briyne, N., Atkinson, J., Pokludová, L., Borriello, S.P., Price, S., 2013. Factors influencing antibiotic prescribing habits and use of sensitivity testing amongst veterinarians in Europe. Vet. Rec. 173, 475.

Dorado-Garcia, A., Mevius, D.J., Jacobs, J.J., Van Geijlswijk, I.M., Mouton, J.W., Wagenaar, J.A., Heederik, D.J., 2016. Quantitative assessment of antimicrobial resistance in livestock during the course of a nationwide antimicrobial use reduction in the Netherlands. J. Antimicrob. Chemother. 71, 3607-3619.

EMA, 2013. Revised ESVAC Reflection Paper on Collecting Data on Consumption of Antimicrobial Agents Per Animal Species, on Technical Units of Measurement and Indicators for Reporting Consumption of Antimicrobial Agents in Animals. Available. . https://www.ema.europa.eu/en/documents/scientific-guideline/ revised-european-surveillance-veterinary-antimicrobial-consumption-esvacreflection-paper-collecting_en.pdf.

Guardabassi, L., Prescott, J.F., 2015. Antimicrobial stewardship in small animal veterinary practice: from theory to practice. Vet. Clin. North Am. Small Anim. Pract. 45, 361-376 vii.

Hardefeldt, L.Y., Browning, G.F., Thursky, K., Gilkerson, J.R., Billman-Jacobe, H., Stevenson, M.A., Bailey, K.E., 2017. Antimicrobials used for surgical prophylaxis by companion animal veterinarians in Australia. Vet. Microbiol. 203, 301-307.

Hardefeldt, L.Y., Gilkerson, J.R., Billman-Jacobe, H., Stevenson, M.A., Thursky, K., Bailey,
K.E., Browning, G.F., 2018a. Barriers to and enablers of implementing antimicrobial stewardship programs in veterinary practices. J. Vet. Intern. Med. 32, 1092-1099.

Hardefeldt, L.Y., Selinger, J., Stevenson, M.A., Gilkerson, J.R., Crabb, H., Billman-Jacobe, H., Thursky, K., Bailey, K.E., Awad, M., Browning, G.F., 2018b. Population wide assessment of antimicrobial use in dogs and cats using a novel data source - a cohort study using pet insurance data. Vet. Microbiol. 225, 34-39.

Hopman, N.E.M., Hulscher, M.E.J.L., Graveland, H., Speksnijder, D.C., Wagenaar, J.A., Broens, E.M., 2018. Factors influencing antimicrobial prescribing by Dutch companion animal veterinarians: a qualitative study. Prev. Vet. Med. 158, 106-113.

Hulscher, M.E.J.L., Grol, R.P.T.M., Van der Meer, J.W.M., 2010. Antibiotic prescribing in hospitals: a social and behavioural scientific approach. Lancet Infect. Dis. 10, $167-175$.

Jaarsma, D.A., Dolmans, D.H., Scherpbier, A.J., Van Beukelen, P., 2008. Preparation for practice by veterinary school: a comparison of the perceptions of alumni from a traditional and an innovative veterinary curriculum. J. Vet. Med. Educ. 35, 431-438.

Jessen, L.R., Sorensen, T.M., Lilja, Z.L., Kristensen, M., Hald, T., Damborg, P., 2017. Cross-sectional survey on the use and impact of the Danish national antibiotic use guidelines for companion animal practice. Acta Vet. Scand. 59 81-017-0350-8.

King, C., Smith, M., Currie, K., Dickson, A., Smith, F., Davis, M., Flowers, P., 2018. Exploring the behavioural drivers of veterinary surgeon antibiotic prescribing: a qualitative study of companion animal veterinary surgeons in the UK. BMC Vet. Res. 14 332-018-1646-2.

KNMvD (Royal Netherlands Veterinary Association), 2013. Aantal Zelfstandige Dierenartsenpraktijken in Nederland. databank KNMvD 24 juni.

Linting, M., Van der Kooij, A., 2012. Nonlinear principal components analysis with CATPCA: a tutorial. J. Pers. Assess. 94, 12-25.

Mateus, A.L., Brodbelt, D.C., Barber, N., Stark, K.D., 2014. Qualitative study of factors associated with antimicrobial usage in seven small animal veterinary practices in the UK. Prev. Vet. Med. 117, 68-78.

McEwen, S.A., Collignon, P.J., 2018. Antimicrobial resistance: a one health perspective. Microbiol. Spectr. 6. https://doi.org/10.1128/microbiolspec.ARBA-0009-2017.

NETHMAP/MARAN, 2018. NethMap: Consumption of Antimicrobial Agents and Antimicrobial Resistance Among Medically Important Bacteria in the Netherlands/ MARAN: Monitoring of Antimicrobial Resistance and Antibiotic Usage in Animals in the Netherlands in 2017. Available. https://www.rivm.nl/bibliotheek/rapporten/ 2018-0046.pdf.

Prescott, J.F., Boerlin, P., 2016. Antimicrobial use in companion animals and good stewardship practice. Vet. Rec. 179, 486-488.

Smith, M., King, C., Davis, M., Dickson, A., Park, J., Smith, F., Currie, K., Flowers, P., 2018. Pet owner and vet interactions: exploring the drivers of AMR. Antimicrob. Resist. Infect. Control 7 46-018-0341-1. eCollection 2018.

Speksnijder, D.C., Graveland, H., Eijck, I.A.J.M., Schepers, R.W.M., Heederik, D.J.J., Verheij, T.J.M., Wagenaar, J.A., 2017. Effect of structural animal health planning on antimicrobial use and animal health variables in conventional dairy farming in the Netherlands. J. Dairy Sci. 100, 4903-4913.

Speksnijder, D.C., Jaarsma, D.A., Verheij, T.J., Wagenaar, J.A., 2015. Attitudes and perceptions of Dutch veterinarians on their role in the reduction of antimicrobial use in farm animals. Prev. Vet. Med. 121, 365-373.

Spellberg, B., Bartlett, J.G., Gilbert, D.N., 2013. The future of antibiotics and resistance. N. Engl. J. Med. 368, 299-302.

STAATSCOURANT, 2013. Regeling van de staatssecretaris van economische zaken van 14 januari 2013, nr. WJZ / 13002270, houdende wijziging van de regeling diergeneesmiddelen in verband met het uitvoeren van een gevoeligheidsbepaling bij toepassing of aflevering van bepaalde diergeneesmiddelen.

Werkgroep Veterinair Antibiotica Beleid (Working Party for Policy on Veterinary Antimicrobials), 2019. WVAB-richtlijn classificatie van veterinaire antimicrobiële middelen. Available. https://www.wvab.nl/. 